\title{
BMJ Open Prospective multicentre randomised clinical trial comparing survival rates, quality of life and nutritional status between advanced gastric cancer patients with different follow-up intensities: study protocol for the STOFOLUP trial
}

\begin{abstract}
Bang Wool Eom (D) , ${ }^{1}$ Dong-Hoe Koo, ${ }^{2}$ Ji Yeong An, ${ }^{3}$ Han Hong Lee, ${ }^{4}$ Hyoung-II Kim (D) , ${ }^{5}$ Hoon Hur, ${ }^{6}$ Moon-Won Yoo, ${ }^{7}$ Min-Hee Ryu, ${ }^{8}$ Hyuk-Joon Lee, ${ }^{9}$ Su Mi Kim, ${ }^{10} \mathrm{Ji}-\mathrm{Ho}$ Park, ${ }^{11}$ Jae Seok Min, ${ }^{12}$ Kyung Won Seo, ${ }^{13}$ Sang-Ho Jeong, ${ }^{14}$ Oh Jeong, ${ }^{15}$ Oh Kyoung Kwon, ${ }^{16}$ Seung Wan Ryu, ${ }^{17}$ Chang Hak Yoo, ${ }^{18}$ Jae Moon Bae, ${ }^{3}$ Keun Won Ryu (D) ${ }^{1}$
\end{abstract}

\begin{abstract}
To cite: Eom BW, Koo D-H, An JY, et al. Prospective multicentre randomised clinical trial comparing survival rates, quality of life and nutritional status between advanced gastric cancer patients with different follow-up intensities: study protocol for the STOFOLUP trial. BMJ Open 2021;11:e056187. doi:10.1136/ bmjopen-2021-056187

- Prepublication history for this paper is available online. To view these files, please visit the journal online (http://dx.doi. org/10.1136/bmjopen-2021 056187).
\end{abstract}

Received 10 August 2021 Accepted 09 November 2021

Check for updates

(c) Author(s) (or their employer(s)) 2021. Re-use permitted under CC BY-NC. No commercial re-use. See rights and permissions. Published by BMJ.

For numbered affiliations see end of article.

Correspondence to

Keun Won Ryu;

docryu@ncc.re.kr

\section{ABSTRACT}

Introduction Patients who underwent curative gastrectomy for gastric cancer are regularly followed-up for the early detection of recurrence and postoperative symptom management. However, there is a lack of evidence with regard to proper surveillance intervals and diagnostic tools. This study aims to evaluate whether frequent surveillance tests have a survival benefit or improve the quality of life in patients who underwent curative resection for advanced gastric cancer.

Methods and analysis The STOFOLUP trial is an investigator-initiated, parallel-assigned, multicentre randomised controlled trial involving 16 hospitals in the Republic of Korea. Patients $(n=886)$ diagnosed with pathological stage II or III gastric adenocarcinoma will be randomised to either the 3-month or the 6-month group at a 1:1 ratio, stratified by trial site and tumour stage. Patients allocated to the 3-month group will undergo an abdominal CT scan every 3 months postoperatively and those allocated to the 6-month group will undergo CT every 6 months. The primary endpoint is 3-year overall survival and the secondary endpoints are quality of life, as assessed using KOrean QUality of life in Stomach cancer patients Study group-40, the European Organization for Research and Treatment of Cancer Quality of Life Questionnaire Core 30 and the stomach cancer-specific module (ST022), and nutritional outcomes. Other survival data including data concerning 3-year disease-free survival, recurrence-free survival, gastric cancer-specific survival and postrecurrence survival will also be estimated. The first patient was enrolled on July 2021 and active patient enrolment is currently underway.

Ethics and dissemination This study has been approved by the Institutional Review Board of eight of the participating hospitals (NCC 2021-0085,

\section{Strengths and limitations of this study}

- The STOFOLUP trial is the first multi-centre, prospective randomised trial to determine an effective surveillance interval after curative gastrectomy for advanced gastric cancer.

- The results of this trial will provide high-level evidence on whether frequent surveillance is associated with a survival benefit and improves quality of life and nutritional outcomes.

- This trial will collect comprehensive data, including various nutritional parameters, endoscopic findings and postgastrectomy symptoms.

- The follow-up interval can be modified during adjuvant chemotherapy, which can diminish the differences between the two groups.

- Detailed management methods for postgastrectomy symptoms and nutritional support methods are not standardised among participating hospitals.

KBSMC2021-01-059, SMC 2021-01-140, KC210EDE0082, 4-2021-0281, AJIRB-MED-INT-20-608, 2021-0515 and H2102-093-1198). This study will be disseminated through peer-reviewed publications, national or international conferences.

Trial registration number NCT04740346.

\section{INTRODUCTION}

Patients who underwent curative gastrectomy for gastric cancer are regularly followed-up for several reasons. The first goal is early detection of recurrence of the primary tumour and timely treatment. The second goal is to 
find a secondary cancer in the remnant stomach or any other organ. The third goal is to manage postgastrectomy symptoms and nutritional problems, and the final one is to provide exact information about the disease and provide psychological support. For these reasons, regular surveillance has been performed for several years postoperatively.

Although this regular surveillance has been commonly performed worldwide, there is a lack of evidence with regard to proper surveillance interval and diagnostic tools after curative gastrectomy for gastric cancer. In National Comprehensive Cancer Network guidelines, indefinite intervals and indications have been described, for example, physical examination every 3-6 months for $1-2$ years and then every $6-12$ months for $3-5$ years and CT every 6-12 months for the first 2 years and then annually up to 5 years. ${ }^{1}$ Moreover, there is no recommended interval for laboratory testing and upper gastrointestinal endoscopy, and they have only described these as clinically indicated. In Japanese gastric cancer treatment guidelines, physical examination, laboratory test including the test for tumour markers is specified to be conducted every 3 months, and CT scan every 6 months for the first 2 years. ${ }^{2}$ However, the guideline clearly demonstrates that evidence is lacking and it is impossible to make any recommendation owing to the paucity of prospective studies of follow-up programmes. In the Korean practice guideline for gastric cancer, which was organised based on the level of evidence and grading of recommendations, there is no statement concerning postoperative surveillance. ${ }^{3}$

The lack of evidence emphasises the need for a prospective trial supporting an effective strategy for a surveillance programme. In this study, we intend to determine whether frequent surveillance tests result in a survival benefit or improvement of quality of life (QoL) in patients who underwent curative resection for locally advanced gastric cancer. Thus, we planned a randomised controlled trial to compare the overall survival (OS) rates, QoL and nutritional status between patients who have been followed-up every 3 months (3-month group) and those who have been followed-up every 6 months (6-month group) after curative gastrectomy for gastric cancer.

\section{METHODS AND ANALYSIS}

\section{Study design}

The STOFOLUP trial is an investigator-initiated, parallelassigned, multicentre randomised controlled trial comparing clinical outcomes according to the follow-up interval in patients who underwent radical gastrectomy for advanced gastric cancer. Eligible patients will be randomly allocated to either 3-month or 6-month group and survival rates, QoL and nutritional outcomes will be compared between them. The protocol for this study was developed by researchers of eight institutions; National Cancer Center, Kangbuk Samsung Hospital, Samsung Medical Center, Seoul St. Mary's Hospital, Yonsei University Severance Hospital, Ajou University Hospital, Asan
Medical Center and Seoul National University Hospital, and finally, a total of 16 institutions in the Republic of Korea will be involved in this trial.

This study has so far been approved by the Institutional Review Board of eight of the participating hospitals (National Cancer Center (NCC 2021-0085), Kangbuk Samsung Hospital (KBSMC2021-01-059), Samsung Medical Center (SMC 2021-01-140), Seoul St. Mary's Hospital (KC21OEDE0082), Yonsei University Severance Hospital (4-2021-0281), Ajou University Hospital (AJIRBMED-INT-20-608), Asan Medical Center (2021-0515) and Seoul National University Hospital (H-2102-093-1198)). Written informed consent will be obtained from all participants before recruitment, and the study will be conducted in accordance with the principles of the Declaration of Helsinki and Good Clinical Practice Guidelines. This trial has been registered in the database of clinical trials (NCT01804998).

The first patient was enrolled on 5 July 2021 and active patient enrolment is currently underway. The estimated study completion date is 30 June 2026.

\section{Study population}

Participants will be recruited at each participating hospital consecutively. A patient scheduled for curative gastrectomy for clinical stage II or III gastric cancer can be a candidate of this trial. Physicians will explain the overview of this trial to candidates before operation, and candidates who agree to participate in this trial will sign the informed consent form. Radical gastrectomy with D2 lymph node dissection will be performed according to the gastric cancer treatment guidelines as scheduled. Enrolment will be conducted after pathological results are reported, and inclusion and exclusion criteria will be as follows.

\section{Inclusion criteria}

1. Patients aged 19 years or more.

2. Patients who are diagnosed with pathological stage II or III gastric adenocarcinoma according to the American Joint Committee on Cancer staging system eighth edition. ${ }^{4}$

3. Patients who can understand all information about the trial and decide for themselves whether to participate in this study.

\section{Exclusion criteria}

1. Vulnerable patients (eg, pregnant or lactating women and individuals with intellectual disability).

2. Patients who cannot undergo CT owing to poor kidney function or severe adverse effects.

3. Patients who are already in another study and cannot follow the schedule for this trial.

4. Patients who are diagnosed with a cancer other than gastric cancer within 5 years before the gastrectomy.

5. Patients being treated for a cancer other than gastric cancer. 


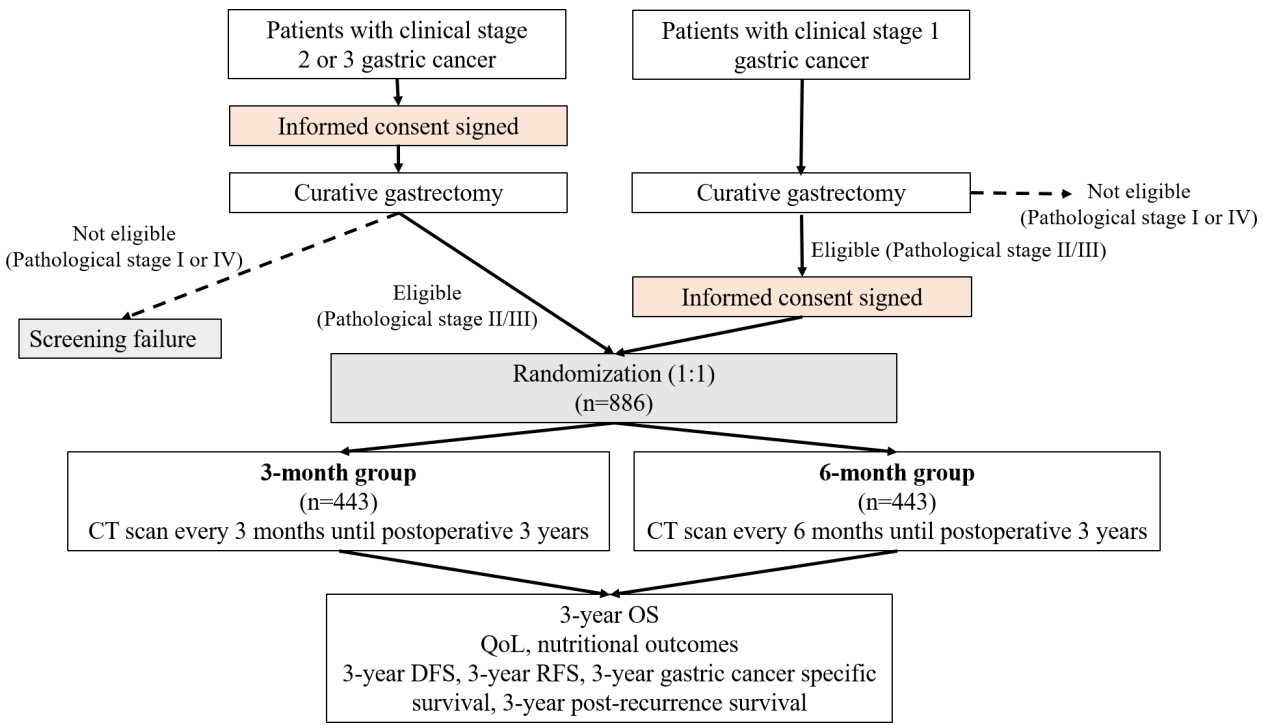

Figure 1 Study flow. DFS, disease-free survival; QoL, quality of life; RFS, recurrence-free survival.

\section{Drop-out criteria}

Patients who meet the following criteria will be dropped out from this trial.

1. Patients who ask to withdraw from this trial.

2. Patients whose data are insufficient because they have not visited four or more times on the scheduled date.

3. Patients who cannot follow the schedule at any point during the study period at the judgement of a responsible researcher (physicians).

When a patient who has signed the informed consent before the operation meets the eligibility criteria based on the pathological report, he/she will be enrolled in this trial. In contrast, a physician can also introduce this trial to a patient who was not informed of this trial before operation but was diagnosed with pathological stage II or III gastric cancer postoperatively. The patient can be enrolled in this trial after signing the informed consent; however, he/she will be excluded from the QoL analysis because of the absence of preoperative (baseline) QoL data. Figure 1 schematically describes the study flow.

\section{Randomisation}

A web-based clinical trial management system (iCReaT; Korea National Institute of Health, Cheongju, Republic of Korea: icreat.nih.go.kr) will be used for randomisation. Eligible patients will be registered in this system and randomly allocated to either the 3-month or the 6-month group at a 1:1 ratio. The permuted block randomisation will be used to generate the initial randomisation sequence, stratified by trial site and tumour stage (pathological stage II vs III).

\section{Interventions}

After randomisation, patients allocated to the 3-month group will be followed-up every 3 months until postoperative 3 years (table 1). At each visit, the body weight, laboratory test parameters, including serum iron, vitamin $\mathrm{B}_{12}$ and tumour markers, abdominal CT scan and chest
X-ray will be performed as scheduled. Data on prescription history for gastrointestinal symptom-relief and iron and vitamin $B_{12}$ supplements will also be collected. Esophagogastroduodenoscopy (EGD) and body composition analysis will be performed annually, and QoL questionnaire survey will be administered preoperatively, and at postoperative 1, 6, 12, 24 and 36 months.

Patients allocated to the 6-month group will visit the hospital every 6 months, with the diagnostic tests and intervals for EGD, body composition analysis and QoL survey being the same as those in the 3-month group.

During adjuvant chemotherapy, the follow-up schedule can be modified at the discretion of the responsible physician. Additional hospital visits and diagnostic tests are allowed for patient safety and symptom management. Moreover, unscheduled diagnostic examination for other diseases is permitted in patient with symptoms; these patients should be treated as needed.

When recurrence is suspected during a scheduled test such as CT and EGD, further diagnostic tests with frequent visits are expected. Thus, patients with suspicious recurrent lesion will not follow the set schedule after this, and only survival data will be collected for them.

\section{Outcome measures}

The primary endpoint is 3-year OS rate. The OS will be estimated from the operation day to the date of death or last follow-up.

The key secondary endpoints are QoL and nutritional outcomes. QoL will be assessed using three types of questionnaires; the KOrean QUality of life in Stomach cancer patients Study group-40 (KOQUSS-40), the European Organization for Research and Treatment of Cancer (EORTC) Quality of Life Questionnaire (QLQ) Core 30 (C30), and the stomach cancer-specific module (EORTC QLQ STO22)..$^{5-7}$ KOQUSS-40 is a symptom-focused questionnaire for patients undergoing gastrectomy for 


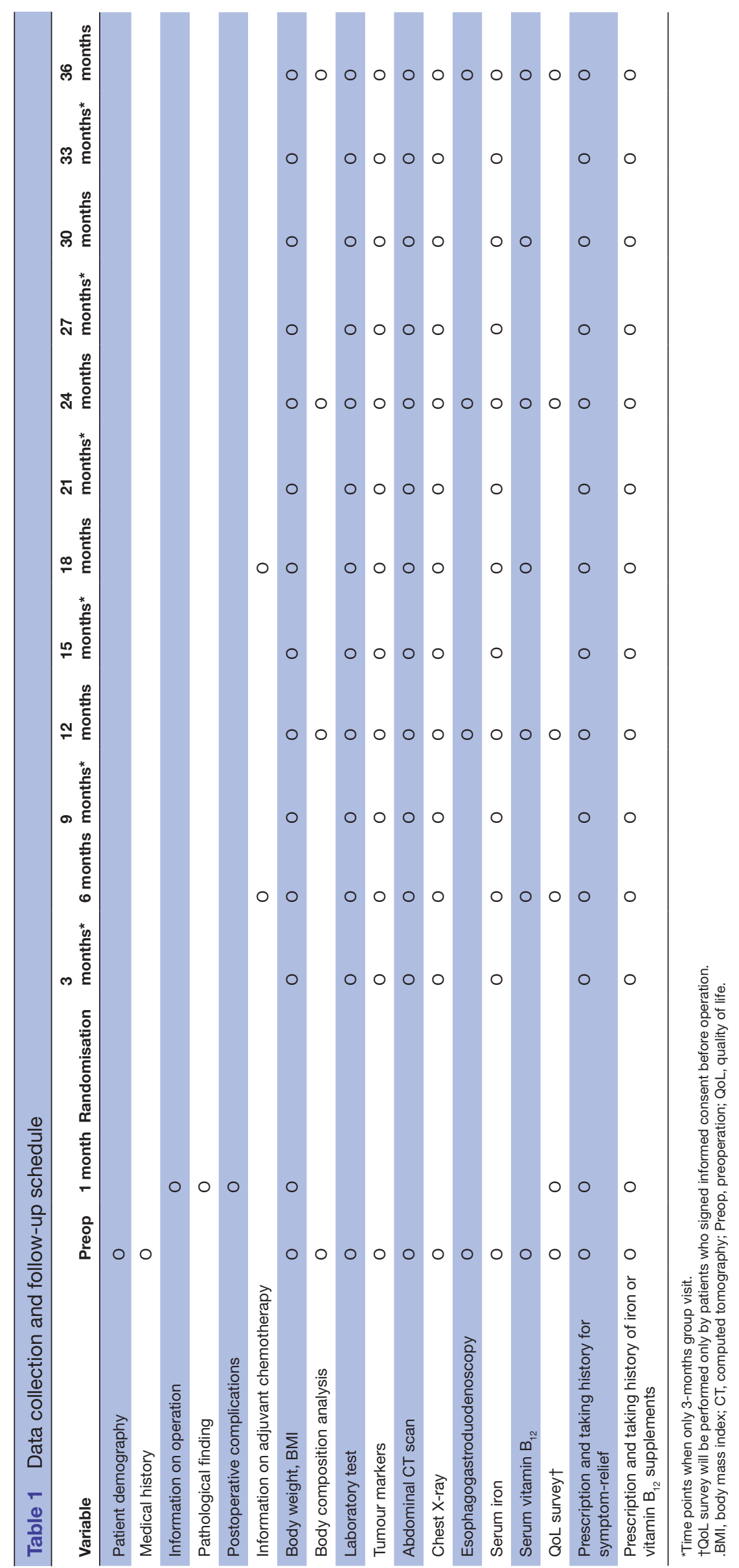

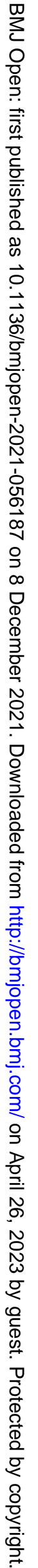


gastric cancer. It consists of 40 items in 11 domains and its scoring ranges from 0 (the worst) to 100 (the best) for each domain. The summary score for KOQUSS-40, defined as the mean of eight equally weighted symptom domains, will be calculated in each case. The validated Korean version of the EORTC QLQ-C30 and STO22 are composed of 30 items in 15 scales and 22 items in 10 scales, respectively. Scoring ranges from 0 (the worst) to 100 (the best) for functional scales and from 0 (the best) to 100 (the worst) for symptom scales. The summary scores of EORTC QLQ-C30 and STO22 will also be calculated to evaluate patients' overall QoL. ${ }^{8}{ }^{9}$ Nutritional outcomes include changes in body weight, body mass index, body composition analysis, serum haemoglobin, albumin, protein, iron, total iron binding capacity and vitamin $\mathrm{B}_{12}$ over time.

The 3-year disease-free survival (DFS), 3-year recurrencefree survival (RFS), 3-year gastric cancer specific survival and 3-year postrecurrence survival are also included as secondary endpoints. DFS will be calculated from the operation day to the date of event occurrence or date of last follow-up. The events for DFS include recurrence of the primary tumour at the resection margin or a distant organ, metachronous cancer in the remnant stomach, newly developed cancer in another organ and death from any cause. For RFS, the events include recurrence of the primary tumour at the resection margin or a distant organ and death from any cause. The postrecurrence survival is defined as the time from recurrence to the date of death from any cause or last follow-up.

In addition, subgroup analyses will be conducted by pathological stage (stage II vs III), extent of gastrectomy (distal vs total gastrectomy) and adjuvant chemotherapy (received vs not received). Each endpoint will be compared between the 3-month and 6-month groups in specific subgroups, and favourable follow-up intensity for each subgroup will be determined.

\section{Data management and monitoring}

Data will be collected as an electronic case report form (eCRF) and managed in a web-based platform (iCReaT; Korea National Institute of Health, Cheongju, Republic of Korea). Clinical research coordinators of each of our site will be trained in using iCReaT system before initiating data entry and will enter the data according to standardised data entry guidelines. Data management will be coordinated by the Ajou University Medical Center Clinical Trial Center. Regular review of eCRFs will be performed by the data management team and all errors will be sent out to each site as queries. Regular data monitoring for each site will also be conducted.

An independent data monitoring committee (IDMC) will be organised by independent experts who will not participate in this trial. IDMC will monitor the overall process of the trial including safety data.

\section{Sample size calculation}

The hypothesis of the poSTOperative FOLlow UP of gastric cancer patients for improved survival and quality of life
(STOFOLUP) trial is that the 3-year OS will be different between the 3-month and 6-month groups. We assumed that the 3-year OS rate in the 6-month group will be $83 \%$ based on the results of the Capecitabine and Oxaliplatin Adjuvant Study in Stomach Cancer (CLASSIC) trial. ${ }^{10}$ Moreover, a survival difference of $6 \%$ has been considered between the two groups based on the COLOFOL trial. ${ }^{11}$ The sample size is planned for an accrual period of 24 months and a follow-up duration of 36 months. A total of 796 events are needed to detect this difference with an alpha error of 0.05 and a statistical power of 0.80 . A drop-out rate of $10 \%$ has been considered and the final sample size is estimated to be 443 patients in each group (886 patients in total).

\section{Statistical analysis}

Both intention-to-treatment (ITT) analysis and perprotocol analysis will be conducted, and the primary analysis will be performed in the ITT population. The survival rates will be estimated using the Kaplan-Meier method and the differences between survival curves will be tested using the log-rank test. The Cox proportional hazards model will also be used to calculate the HR with $95 \%$ CI.

The QoL scores are non-normally distributed and differences in QoL between the two groups at baseline or any specific time point will be assessed via the non-parametric Wilcoxon rank sum test. The differences between overall QoL scores over time will be evaluated using a linear mixed effect model. Nutritional outcomes will be determined using repeatedly collected continuous variables such as QoL scores. Student's t-test and a linear mixed effect model will be used for cross-sectional comparison and for longitudinal comparison, respectively.

Regarding other descriptive statistics, categorical variables will be analysed by $\chi^{2}$ test or Fisher's exact test and continuous variables by Student's t-test or Wilcoxon rank sum test.

Statistical analyses will be performed using SAS V.9.4 (SAS Institute, Cary, NC). P values $<0.05$ will be considered statistically significant.

\section{Patient and public involvement}

No patients or members of the public were involved in the design, or conduct, or reporting or dissemination plans of this research.

\section{DISCUSSION}

The STOFOLUP trial is an investigator initiated, randomised controlled trial involving 16 institutions in Korea, and the first large-scale prospective trial with regard to surveillance after curative gastrectomy for gastric cancer, worldwide. In this trial, we will determine whether frequent surveillance tests are associated with survival benefits or improvement of QoL in patients with gastric cancer. The results of this trial will provide highlevel evidence and will affect actual follow-up schedules in clinical practice. 
Several retrospective studies on surveillance after curative gastrectomy for gastric cancer have been reported. In a review article analysing nine retrospective studies, six of nine studies compared patients with symptomatic recurrence with those with asymptomatic recurrence. ${ }^{12}$ All six studies showed significantly increased postrecurrence survival in patients with asymptomatic recurrence, which can be entirely explained by lead-time bias. ${ }^{13-18}$ Two of six studies showed that the OS duration was longer in the asymptomatic groups; however, no significant difference was observed in the other four studies. ${ }^{15-18}$ Another three studies comparing OS between intensive and less intensive surveillance failed to figure out any difference in OS. ${ }^{91920}$ Thus, oncological benefits of intensive surveillance have not been proven in previous retrospective studies.

Although there is no well-designed prospective study for patients with gastric cancer to date, a largescaled randomised clinical trial comparing more frequent follow-up testing with less follow-ups has been performed in patients with stage II or III colorectal cancer (COLOFOL trial) ${ }^{11}$ A total of 2509 patients from 24 hospitals were included in this study, and patients were randomly assigned to the high-intensity or the lowintensity group. The study set an absolute difference in mortality rate of $6 \%$ between the high-intensity and lowgroups, and the hypothesis was that intensive follow-up testing would decrease mortality rates. In its final analysis, frequent follow-up testing did not produce a significant rate reduction in the 5 -year overall mortality and colorectal cancer-specific mortality.

The present study was designed with reference to COLOFOL trial with regard to the difference in OS rate between the two groups, and the follow-up interval was decided by referring to a previous nationwide survey study. ${ }^{11}{ }^{21}$ In this previous nationwide survey study, 205 members of the Korean Gastric Cancer Association participated, and a majority of responders indicated the execution of follow-up examinations every 3 or 6 months for the first year and every 6 months for the next 4 years in patients with advanced gastric cancer. Most responders $(88.5 \%)$ used CT as the imaging modality to detect recurrence. Thus, we planned to conduct a comparison between the 3-month and the 6-month groups.

Short-interval surveillance is associated with early recurrence detection; consequently, DFS can be affected by lead-time bias. Therefore, the primary endpoint was decided as OS in this study. We will also evaluate postrecurrence survival, which has been reported to be longer in patients with asymptomatic recurrence than in those with symptomatic recurrence in previous studies. ${ }^{13-15} 1718$ Postrecurrence survival can also be associated with leadtime bias; however, it would be an important outcome with regard to prognosis.

QoL is one of the most important clinical outcomes in cancer patients. Timely and appropriate symptom management can improve QoL, which is another key purpose of regular surveillance. In this study, KOQUSS-40 will be used to assess the QoL. ${ }^{5}$ Frequent follow-up indicates frequent counselling, education and prescription, and these delicate management strategies help patients in self-controlling their symptoms, resulting in better physical, social and emotional functions. KOQUSS-40 focuses on postgastrectomy symptoms and we will evaluate whether there is a significant difference in KOQUSS-40 questionnaire scores in individuals with different follow-up intervals. Moreover, we will determine if KOQUSS-40 can discern the severity of symptoms and the degree of QoL better than the conventional QoL questionnaires: EORTC QLQ-C30 and STO22.

Nutritional status after gastrectomy has been evaluated in many previous studies. ${ }^{22-27}$ Postoperative severe weight loss is associated with a higher mortality rate, and patients who undergo total gastrectomy had been reported to have worse QoL than those who undergo distal gastrectomy. ${ }^{22-25} \mathrm{~A}$ considerable number of patients experience iron deficiency and most patients who undergo total gastrectomy have vitamin $\mathrm{B}_{12}$ deficiency. ${ }^{26}{ }^{27}$ Frequent follow-ups may lead to frequent counselling of eating restrictions and a close monitoring of nutritional deficiencies. The effect of follow-up interval on nutritional status is another important endpoint of this study.

Frequent CT scans augment exposure to radiation and increase the risk of adverse effects of contrast media. The effective dose for single abdominal CT is approximately 10-15 mSv. Considering that the effective dose limit for radiation workers is $100 \mathrm{mSv}$ for 5 years in the Republic of Korea, reducing the frequency of CT scans as much as possible is necessary. Although the incidence is very low, severe adverse effects of contrast media, for example, anaphylaxis shock, must be considered as well. Thus, the results of this study can provide important evidence for risks and benefits depending on the CT follow-up interval.

This study has some limitations. First, the follow-up interval can be modified during adjuvant chemotherapy, which can diminish the differences between the two groups. Physical examination, laboratory tests and prescriptions for adverse effects of chemotherapy are not limited during adjuvant chemotherapy and additional CT checks for oncological evaluation are also allowed during adjuvant chemotherapy. Second, detailed management methods for postgastrectomy symptoms and nutritional support methods are not standardised among participating hospitals. In practice, the severity of symptoms cannot be classified into standard categories and its management strategy widely varies. Each physician and hospital have their own strategy or policy. Thus, a distinct management procedure will be conducted in each clinic and ideally, the same strategy should be followed between two groups. Third, the planned follow-up period is 3 years and there is a limitation of long-term results beyond 3 years in this study. Although most recurrence occurs 
within 3 years after operation and symptoms and QoL improve after 2 years postoperatively, development of secondary cancer or changes in nutritional status can take longer. Data concerning secondary cancer development or long-term nutritional status evaluation are limited in this study. ${ }^{9} 16$

In conclusion, the STOFOLUP trial is the first largescaled multicentre randomised controlled trial to evaluate oncological and clinical effect of postoperative surveillance in patients who underwent curative resection for advanced gastric cancer. We hope that the results of this trial can provide confirmative evidence for appropriate surveillance intervals and diagnostic tools in cases of advanced gastric cancer.

\section{Author affiliations}

${ }^{1}$ Center for Gastric Cancer, National Cancer Center, Goyang, The Republic of Korea ${ }^{2}$ Department of Internal Medicine, Kangbuk Samsung Hospital, Sungkyunkwan University School of Medicine, Seoul, The Republic of Korea

${ }^{3}$ Department of Surgery, Samsung Medical Center, Sungkyunkwan University School of Medicine, Seoul, The Republic of Korea

${ }^{4}$ Department of Surgery, Seoul St. Mary's Hospital, College of Medicine, The Catholic University of Korea, Seoul, The Republic of Korea

${ }^{5}$ Department of Surgery, Yonsei University Severance Hospital, Yonsei University School of Medicine, Seoul, The Republic of Korea

${ }^{6}$ Department of Surgery, Ajou University Hospital, Ajou University School of Medicine, Suwon, The Republic of Korea

${ }^{7}$ Department of Surgery, Asan Medical Center, University of Ulsan College of Medicine, Seoul, The Republic of Korea

${ }^{8}$ Department of Oncology, Asan Medical Center, University of Ulsan College of Medicine, Seoul, The Republic of Korea

${ }^{9}$ Department of Surgery and Cancer Research Institute, Seoul National University Hospital, Seoul National University of College of Medicine, Seoul, The Republic of Korea

${ }^{10}$ Department of Surgery, CHA Bundang Medical Center, CHA University, Seongnam, The Republic of Korea

${ }^{11}$ Department of Surgery, Gyeongsang National University Hospital, Gyeongsang National University School of Medicine, Jinju, The Republic of Korea

${ }^{12}$ Department of Surgery, Dongnam Institute of Radiological and Medical Sciences, Busan, The Republic of Korea

${ }^{13}$ Department of Surgery, Kosin University Gospel Hospital, Kosin University College of Medicine, Busan, The Republic of Korea

${ }^{14}$ Department of Surgery, Gyeongsang National University Changwon Hospital, Gyeongsang National University School of Medicine, Changwon, The Republic of Korea

${ }^{15}$ Department of Surgery, Chonnam National University Hwasun Hospital, Chonnam National University Medical School, Hwasun-gun, The Republic of Korea

${ }^{16}$ Department of Surgery, Kyungpook National University Chilgok Hospital, Kyungpook National University School of Medicine, Daegu, The Republic of Korea

${ }^{17}$ Department of Surgery, Keimyung University Dongsan Hospital, Keimyung University School of Medicine, Daegu, The Republic of Korea

${ }^{18}$ Department of Surgery, Kangbuk Samsung Hospital, Sungkyunkwan University School of Medicine, Seoul, The Republic of Korea

Acknowledgements The authors would like to thank Editage (www.editage.co.kr) for English language editing.

Contributors KWR is the grant holder. BWE, D-HK, JYA, HHL, HK, HH, M-HR $\mathrm{H}-\mathrm{JL}$ and KWR participated in the design of the study. BWE, D-HK, JYA, HHL, HK, HH, M-WY, M-HR, H-JL, SMK, J-HP, JSM, KWS, S-HJ, OJ, OKK, S-WR, CHY, JMB and KWR are responsible for conducting the trial as well as patient recruitment. BWE prepared the manuscript. All authors read and approved the final manuscript.

Funding This research was supported by a grant of the Korea Health Technology R\&D Project through the Korea Health Industry Development Institute (KHIDI) (grant number: HI19C0481, HC20C0155), funded by the Ministry of Health \& Welfare, Republic of Korea.
Competing interests None declared.

Patient and public involvement Patients and/or the public were not involved in the design, or conduct, or reporting or dissemination plans of this research.

Patient consent for publication Not required.

Provenance and peer review Not commissioned; externally peer reviewed.

Open access This is an open access article distributed in accordance with the Creative Commons Attribution Non Commercial (CC BY-NC 4.0) license, which permits others to distribute, remix, adapt, build upon this work non-commercially, and license their derivative works on different terms, provided the original work is properly cited, appropriate credit is given, any changes made indicated, and the use is non-commercial. See: http://creativecommons.org/licenses/by-nc/4.0/.

\section{ORCID iDs}

Bang Wool Eom http://orcid.org/0000-0002-0332-2051

Hyoung-II Kim http://orcid.org/0000-0002-6134-4523

Keun Won Ryu http://orcid.org/0000-0002-5935-9777

\section{REFERENCES}

1 NCCN. Guidelines version 2.2021 gastric cancer, 2021. (2021 March 9).

2 Japanese Gastric Cancer Association. Japanese gastric cancer treatment guidelines 2018 (5th edition). Gastric Cancer 2021;24:1-21.

3 Guideline Committee of the Korean Gastric Cancer Association (KGCA), Development Working Group \& Review Panel. Korean practice guideline for gastric cancer 2018: an evidence-based, multidisciplinary approach. J Gastric Cancer 2019;19:1-48.

4 TNM. Classification of malignant tumours. Eighth Edition. Chichester, West Sussex, UK; Hoboken, NJ: Wiley-Blackwell, 2017.

5 Eom BW, Lee J, Lee IS, et al. Development and validation of a Symptom-Focused quality of life questionnaire (KOQUSS-40) for gastric cancer patients after gastrectomy. Cancer Res Treat 2021;53:763-772.

6 Aaronson NK, Ahmedzai S, Bergman B, et al. The European organization for research and treatment of cancer QLQ-C30: a quality-of-life instrument for use in international clinical trials in oncology. J Natl Cancer Inst 1993;85:365-76.

7 Blazeby JM, Conroy T, Bottomley A, et al. Clinical and psychometric validation of a questionnaire module, the EORTC QLQ-STO 22, to assess quality of life in patients with gastric cancer. Eur $J$ Cancer 2004;40:2260-8.

8 Giesinger JM, Kieffer JM, Fayers PM, et al. Replication and validation of higher order models demonstrated that a summary score for the EORTC QLQ-C30 is robust. J Clin Epidemiol 2016;69:79-88.

9 Park $\mathrm{CH}$, Park JC, Chung $\mathrm{H}$, et al. Impact of the surveillance interval on the survival of patients who undergo curative surgery for gastric cancer. Ann Surg Oncol 2016;23:539-45.

10 Bang Y-J, Kim Y-W, Yang H-K, et al. Adjuvant capecitabine and oxaliplatin for gastric cancer after D2 gastrectomy (classic): a phase 3 open-label, randomised controlled trial. The Lancet 2012;379:315-21.

11 Wille-Jørgensen P, Syk I, Smedh K, et al. Effect of more vs less frequent follow-up testing on overall and colorectal Cancer-Specific mortality in patients with stage II or III colorectal cancer. JAMA 2018;319:2095-103.

12 Nilsson M. Postgastrectomy follow-up in the West: evidence base, guidelines, and daily practice. Gastric Cancer 2017;20:135-40.

13 Bennett JJ, Gonen M, D'Angelica M, et al. Is detection of asymptomatic recurrence after curative resection associated with improved survival in patients with gastric cancer? J Am Coll Surg 2005;201:503-10.

14 Böhner H, Zimmer T, Hopfenmüller W, et al. Detection and prognosis of recurrent gastric cancer--is routine follow-up after gastrectomy worthwhile? Hepatogastroenterology 2000;47:1489-94.

15 Kodera Y, Ito S, Yamamura Y, et al. Follow-Up surveillance for recurrence after curative gastric cancer surgery lacks survival benefit. Ann Surg Oncol 2003;10:898-902.

16 Eom BW, Ryu KW, Lee JH, et al. Oncologic effectiveness of regular follow-up to detect recurrence after curative resection of gastric cancer. Ann Surg Oncol 2011;18:358-64.

17 Bilici A, Salman T, Oven Ustaalioglu BB, et al. The prognostic value of detecting symptomatic or asymptomatic recurrence in patients with gastric cancer after a curative gastrectomy. J Surg Res 2013;180:e1-9.

18 Lee S-Y, Lee JH, Hwang NC, et al. The role of follow-up endoscopy after total gastrectomy for gastric cancer. European Journal of Surgical Oncology 2005;31:265-9. 
19 Tan IT, So BYJ, . Value of intensive follow-up of patients after curative surgery for gastric carcinoma. J Surg Oncol 2007;96:503-6.

20 Peixoto RD, Lim HJ, Kim H, et al. Patterns of surveillance following curative intent therapy for gastroesophageal cancer. $J$ Gastrointest Cancer 2014; 45:325-33.

21 Hur $\mathrm{H}$, Song KY, Park CH, et al. Follow-up strategy after curative resection of gastric cancer: a nationwide survey in Korea. Ann Surg Oncol 2010;17:54-64.

22 Park YS, Park DJ, Lee Y, et al. Prognostic roles of perioperative body mass index and weight loss in the long-term survival of gastric cancer patients. Cancer Epidemiol Biomarkers Prev 2018;27:955-62.

23 Aoyama T, Sato T, Maezawa Y, et al. Postoperative weight loss leads to poor survival through poor S-1 efficacy in patients with stage II/III gastric cancer. Int J Clin Oncol 2017;22:476-83.
$24 \mathrm{Kim} \mathrm{AR,} \mathrm{Cho} \mathrm{J,} \mathrm{Hsu} \mathrm{Y-J,} \mathrm{et} \mathrm{al.} \mathrm{Changes} \mathrm{of} \mathrm{quality} \mathrm{of} \mathrm{life} \mathrm{in} \mathrm{gastric}$ cancer patients after curative resection: a longitudinal cohort study in Korea. Ann Surg 2012;256:1008-13.

25 Lee SS, Chung HY, Kwon OK, et al. Long-Term quality of life after distal subtotal and total gastrectomy: Symptom- and Behaviororiented consequences. Ann Surg 2016;263:738-44.

26 Lee $\mathrm{JH}$, Hyung WJ, Kim H-I, et al. Method of reconstruction governs iron metabolism after gastrectomy for patients with gastric cancer. Ann Surg 2013;258:964-9.

27 Hu Y, Kim H-I, Hyung WJ, et al. Vitamin B(12) deficiency after gastrectomy for gastric cancer: an analysis of clinical patterns and risk factors. Ann Surg 2013;258:970-5. 